\title{
Algebra isomorphisms between standard operator algebras
}

by

\author{
Thomas Tonev (Missoula, MT) and Aaron Luttman (Potsdam, NY)
}

\begin{abstract}
If $X$ and $Y$ are Banach spaces, then subalgebras $\mathfrak{A} \subset B(X)$ and $\mathfrak{B} \subset$ $B(Y)$, not necessarily unital nor complete, are called standard operator algebras if they contain all finite rank operators on $X$ and $Y$ respectively. The peripheral spectrum of $A \in \mathfrak{A}$ is the set $\sigma_{\pi}(A)=\left\{\lambda \in \sigma(A):|\lambda|=\max _{z \in \sigma(A)}|z|\right\}$ of spectral values of $A$ of maximum modulus, and a map $\varphi: \mathfrak{A} \rightarrow \mathfrak{B}$ is called peripherally-multiplicative if it satisfies the equation $\sigma_{\pi}(\varphi(A) \circ \varphi(B))=\sigma_{\pi}(A B)$ for all $A, B \in \mathfrak{A}$. We show that any peripherally-multiplicative and surjective $\operatorname{map} \varphi: \mathfrak{A} \rightarrow \mathfrak{B}$, neither assumed to be linear nor continuous, is a bijective bounded linear operator such that either $\varphi$ or $-\varphi$ is multiplicative or anti-multiplicative. This holds in particular for the algebras of finite rank operators or of compact operators on $X$ and $Y$ and extends earlier results of Molnár. If, in addition, $\sigma_{\pi}\left(\varphi\left(A_{0}\right)\right) \neq-\sigma_{\pi}\left(A_{0}\right)$ for some $A_{0} \in \mathfrak{A}$ then $\varphi$ is either multiplicative, in which case $X$ is isomorphic to $Y$, or anti-multiplicative, in which case $X$ is isomorphic to $Y^{*}$. Therefore, if $X \nsubseteq Y^{*}$ then $\varphi$ is multiplicative, hence an algebra isomorphism, while if $X \nsubseteq Y$, then $\varphi$ is anti-multiplicative, hence an algebra anti-isomorphism.
\end{abstract}

1. Introduction. Identifying algebra isomorphisms among maps between Banach algebras has attracted considerable interest. For maps presumed from the beginning to be linear it has been an active area of research, particularly for maps that also preserve some non-algebraic structure, the so-called linear preservers (see e.g. [9, 10, 12]). Recall that the spectrum of an operator $A \in B(X)$ is the compact set $\sigma(A)=\left\{\lambda \in \mathbb{C}:(\lambda I-A) \notin B(X)^{-1}\right\}$. Molnár [11] introduced maps $\varphi$ of an algebra into itself that are weakly multiplicative in the sense that

$$
\sigma(\varphi(A) \circ \varphi(B))=\sigma(A B)
$$

for all algebra elements $A, B$, and has shown that any surjective self-map $\varphi$ that satisfies condition (1) is closely related to an algebra isomorphism in the case of $B(X)$, the algebra of all bounded linear operators on a Banach space $X$, or $C(K)$, the algebra of complex-valued, continuous functions on a first-countable compact Hausdorff space $K$. For uniform algebras and

2000 Mathematics Subject Classification: Primary 46H35; Secondary 46H40, 47L10.

Key words and phrases: standard operator algebra, spectrum of an operator, peripheral spectrum, peripherally-multiplicative operator, algebra isomorphism. 
algebras of type $B(X)$ Molnár's result was extended further in various directions (see e.g. $[3,4,5,8,10,13])$. Rather than requiring that such a map multiplicatively preserves the entire spectrum, however, it is also natural to ask whether preserving particular subsets of the spectrum will suffice. The peripheral spectrum $\sigma_{\pi}(A)=\left\{\lambda \in \sigma(A):|\lambda|=\max _{z \in \sigma(A)}|z|\right\}$ of $A$ is the set of spectral values of $A$ of maximum modulus. In [3, 10] Molnár's result is extended to surjective maps $\varphi: \mathfrak{A} \rightarrow \mathfrak{B}$ between two uniform algebras that instead of (1) satisfy the equation

$$
\sigma_{\pi}(\varphi(A) \circ \varphi(B))=\sigma_{\pi}(A B)
$$

for all $A, B \in \mathfrak{A}$. Maps that satisfy (2) are called peripherally-multiplicative. In the settings of uniform algebras and operator algebras of type $B(X)$, related results involving alternative conditions are also considered (e.g. in $[2,3,12,14,15])$. However, for operator algebras other than $B(X)$, and in particular for non-unital algebras or algebras of compact operators, the subject has not been sufficiently studied.

A subalgebra $\mathfrak{A}$ of $B(X)$, not necessarily complete nor unital, is called a standard operator algebra if it contains all rank one operators. For instance, the algebra of finite rank operators, its norm-closure, and the algebra of compact operators on a Banach space are standard operator algebras. In this paper we extend Molnár's result [11, Theorem 1] to peripherally-multiplicative maps $\varphi$ between distinct and not necessarily unital standard operator algebras. In particular, we do not assume that $\varphi$ is linear, nor continuous, nor that it preserves operators' injectivity or surjectivity. The theorems that follow are the primary results of the paper.

Theorem 1. Let $X, Y$ be Banach spaces, $\mathfrak{A} \subset B(X)$ and $\mathfrak{B} \subset B(Y)$ be standard operator algebras, and $\varphi: \mathfrak{A} \rightarrow \mathfrak{B}$ be a surjective map, a priori neither linear nor continuous. If $\varphi$ is peripherally-multiplicative, i.e. satisfies condition (2) for all $A, B \in \mathfrak{A}$, then it is linear, and either $\varphi$ or $-\varphi$ is multiplicative or anti-multiplicative. Therefore, either $\varphi$ or $-\varphi$ is an algebra isomorphism or anti-isomorphism.

TheOREM 2. Let $\varphi$ be as in Theorem 1. If, in addition, $\sigma_{\pi}\left(\varphi\left(A_{0}\right)\right) \neq$ $-\sigma_{\pi}\left(A_{0}\right)$ for some $A_{0} \in \mathfrak{A}$ then $\varphi$ is a bijective bounded linear operator which is either multiplicative, in which case $X$ is isomorphic to $Y$, or antimultiplicative, in which case $X$ is isomorphic to $Y^{*}$. Thus, if $X \neq Y^{*}$ then $\varphi$ is multiplicative, hence an algebra isomorphism, while if $X \supsetneqq Y$, then $\varphi$ is anti-multiplicative, hence an algebra anti-isomorphism.

Recall that an operator $T \in B(X)$ is of rank at most one if the dimension of its range is less than or equal to 1 . Every such operator has the form $x \otimes f$ for some $x \in X$ and $f \in X^{*}$, the dual space of $X$, where $(x \otimes f) y=f(y) x$. 
The set of all operators in $B(X)$ of rank at most one will be denoted by $B_{1}(X)$.

Note that $\sigma(x \otimes f) \subset\{0, f(x)\}$ and $\sigma_{\pi}(x \otimes f)=\{f(x)\}$. It is easy to see that if $A \in B(X)$ and $x \otimes f \in B_{1}(X)$ then

$$
A \circ(x \otimes f)=(A x) \otimes f .
$$

Therefore, $A \circ(x \otimes f)$ is also an operator of rank at most one, and

$$
\sigma_{\pi}(A \circ(x \otimes f))=\{f(A x)\} .
$$

The following lemma gives a criterion for coincidence of two operators in $B(X)$.

Lemma 1 (Identification Lemma). Let $X$ be a Banach space and $A, B \in$ $B(X)$. If $\sigma_{\pi}(A T)=\sigma_{\pi}(B T)$ for every $T \in B_{1}(X)$, then $A=B$.

Proof. Let $T=x \otimes f \in B_{1}(X)$. If $\sigma_{\pi}(A T)=\sigma_{\pi}(B T)$ for all $T \in B_{1}(X)$, then by (4) we have $\{f(A x)\}=\sigma_{\pi}(A T)=\sigma_{\pi}(B T)=\{f(B x)\}$. Since $f \in X^{*}$ is arbitrary, $A x=B x$ for any $x \in X$, and thus $A=B$.

2. Peripherally-multiplicative maps on standard operator algebras. Throughout this section $\mathfrak{A} \subset B(X)$ and $\mathfrak{B} \subset B(Y)$ denote standard operator algebras on Banach spaces $X$ and $Y$, respectively.

Lemma 2. Every peripherally-multiplicative map $\varphi: \mathfrak{A} \rightarrow B(Y)$ on a standard operator algebra $\mathfrak{A}$ is injective.

Proof. Indeed, if $\varphi(A)=\varphi(B)$ for some $A, B \in \mathfrak{A}$, then the peripheral multiplicativity of $\varphi$ yields $\sigma_{\pi}(A T)=\sigma_{\pi}(\varphi(A) \circ \varphi(T))=\sigma_{\pi}(\varphi(B) \circ \varphi(T))=$ $\sigma_{\pi}(B T)$ for every $T \in B_{1}(X)$. The Identification Lemma 1 implies that $A=B$, i.e. $\varphi$ is injective.

The next lemma shows that if $\varphi$ is a peripherally-multiplicative surjective map between standard operator algebras, then $\varphi\left(B_{1}(X)\right)=B_{1}(Y)$, i.e. $\varphi$ preserves the operators of rank at most one.

Lemma 3. Every peripherally-multiplicative surjective map $\varphi: \mathfrak{A} \rightarrow \mathfrak{B}$ preserves the operators of rank at most one.

Proof. We assume that $\operatorname{dim}(X)>1$ since the result obviously holds if $\operatorname{dim}(X)=1$. Since $B_{1}(Y) \subset \mathfrak{B}$, the surjectivity of $\varphi$ implies that for any $T \in$ $B_{1}(Y)$ there exists $S \in \mathfrak{A}$ with $T=\varphi(S)$. We claim that $S$ is of rank at most one. Assume that $\operatorname{rank}(S)>1$, and let $x_{1}, x_{2}$ be two linearly independent elements in the range of $S$. Hence there are linearly independent elements $x_{1}^{\prime}, x_{2}^{\prime}$ in $X$ with $S x_{i}^{\prime}=x_{i}, i=1,2$. By the Hahn-Banach Theorem we can choose linearly independent linear functionals $f_{1}, f_{2}$ in $X^{*}$ with $f_{1}\left(x_{2}\right)=f_{2}\left(x_{1}\right)=0$ and $f_{i}\left(x_{i}\right)=1, i=1,2$. Since the operator $P=x_{1}^{\prime} \otimes f_{1}-x_{2}^{\prime} \otimes f_{2}$ is of rank at 
most two, it belongs to $\mathfrak{A}$. The operator $S P=x_{1} \otimes f_{1}-x_{2} \otimes f_{2}$ also belongs to $\mathfrak{A}$, and moreover $\sigma_{\pi}(S P)=\{-1,1\}$. On the other hand, $T \circ \varphi(P) \in B_{1}(Y)$ since $T \in B_{1}(Y)$, and therefore $\sigma_{\pi}(\varphi(S) \circ \varphi(P))=\sigma_{\pi}(T \circ \varphi(P))$ is a singleton. Thus $\sigma_{\pi}(\varphi(S) \circ \varphi(P)) \neq \sigma_{\pi}(S P)$, in violation of the peripheral multiplicativity of $\varphi$. Consequently, $\operatorname{rank}(S) \leq 1$, i.e. $S \in B_{1}(X)$, and thus $T \in \varphi\left(B_{1}(X)\right)$. Hence, $B_{1}(Y) \subset \varphi\left(B_{1}(X)\right)$.

Since $\mathfrak{B}$ is a standard operator algebra on $Y$ and $\varphi$ is bijective, $\varphi^{-1}$ is also peripherally-multiplicative, and the above arguments applied to $\varphi^{-1}$ yield the opposite inclusion.

Lemma 4. Every peripherally-multiplicative surjective map $\varphi: \mathfrak{A} \rightarrow \mathfrak{B}$ is linear and one of the following holds:

(a) there are bijective linear operators $C: X \rightarrow Y$ and $D: X^{*} \rightarrow Y^{*}$ such that $\varphi(x \otimes f)=C x \otimes D f$ for every $x \in X$ and $f \in X^{*}$, or

(b) there are bijective linear operators $E: X^{*} \rightarrow Y$ and $F: X \rightarrow Y^{*}$ such that $\varphi(x \otimes f)=E f \otimes F x$ for all $x \in X$ and $f \in X^{*}$.

Proof. We first show that under the hypotheses $\varphi$ is a linear operator. If $T=u \otimes g \in B_{1}(Y)$ for some $u \in Y$ and $g \in Y^{*}$, then Lemma 3 implies that $T=\varphi(S)$ for some $S=x \otimes f \in B_{1}(X)$ with $x \in X$ and $f \in X^{*}$. The peripheral multiplicativity of $\varphi$, the linearity of $f$ and $g$ and (4) imply that for all $\lambda, \mu \in \mathbb{C}$ and $A, B \in \mathfrak{A}$ we have

$$
\begin{aligned}
\sigma_{\pi} & (\varphi(\lambda A+\mu B) \circ T)=\sigma_{\pi}(\varphi(\lambda A+\mu B) \circ \varphi(S))=\sigma_{\pi}((\lambda A+\mu B) \circ S) \\
& =\sigma_{\pi}((\lambda A+\mu B) \circ(x \otimes f))=\sigma_{\pi}(((\lambda A+\mu B) x) \otimes f)=\{f((\lambda A+\mu B) x)\} \\
& =\{f(\lambda(A x)+\mu(B x))\}=\{\lambda f(A x)+\mu f(B x)\}=\lambda\{f(A x)\}+\mu\{f(B x)\} \\
& =\lambda \sigma_{\pi}(A S)+\mu \sigma_{\pi}(B S)=\lambda \sigma_{\pi}(\varphi(A) \circ \varphi(S))+\mu \sigma_{\pi}(\varphi(B) \circ \varphi(S)) \\
& =\lambda \sigma_{\pi}(\varphi(A) \circ(u \otimes g))+\mu \sigma_{\pi}(\varphi(B) \circ(u \otimes g)) \\
& =\lambda \sigma_{\pi}((\varphi(A) u) \otimes g)+\mu \sigma_{\pi}((\varphi(B) u) \otimes g) \\
& =\lambda\{g((\varphi(A)) u)\}+\mu\{g((\varphi(B)) u)\}=\{\lambda g((\varphi(A)) u)+\mu g((\varphi(B)) u)\} \\
& =\{g((\lambda \varphi(A)+\mu \varphi(B)) u)\}=\sigma_{\pi}((\lambda \varphi(A)+\mu \varphi(B)) \circ(u \otimes g)) \\
& =\sigma_{\pi}((\lambda \varphi(A)+\mu \varphi(B)) \circ \varphi(S))=\sigma_{\pi}((\lambda \varphi(A)+\mu \varphi(B)) \circ T) .
\end{aligned}
$$

The Identification Lemma 1 implies that $\varphi(\lambda A+\mu B)=\lambda \varphi(A)+\mu \varphi(B)$, i.e. $\varphi$ is linear, as claimed. Consequently, $\varphi$ is a bijective linear operator.

Thus $\varphi$ is linear and preserves the operators of rank at most one by Lemma 3. The characterization of $\varphi$ as being of the form (a) or (b) is well-known in the case when $\mathfrak{A}=B(X), \mathfrak{B}=B(Y)$ and $\varphi$ is a linear spectrum-preserving map. The proof provided in [6, Theorem 2] actually works also for arbitrary standard operator algebras in the case when $\varphi$ preserves operators of rank at most one. 
Observe that, if $\varphi$ is multiplicative or anti-multiplicative and preserves the peripheral spectra of operators (i.e. $\left.\sigma_{\pi}(\varphi(A))=\sigma_{\pi}(A), A \in \mathfrak{A}\right)$, then $\varphi$ is peripherally-multiplicative. Lemmas 2 and 4 imply the following:

COROLlary 1. Every multiplicative [resp. anti-multiplicative] surjective map $\varphi: \mathfrak{A} \rightarrow \mathfrak{B}$ that preserves the peripheral spectra of operators is an algebra isomorphism [resp. anti-isomorphism] onto $\mathfrak{B}$.

The following more general theorem extends one of the main results in $[11]$.

Theorem 1. Let $X, Y$ be Banach spaces, $\mathfrak{A} \subset B(X)$ and $\mathfrak{B} \subset B(Y)$ be standard operator algebras, and $\varphi: \mathfrak{A} \rightarrow \mathfrak{B}$ be a surjective map. If $\varphi$ is peripherally-multiplicative, i.e. $\sigma_{\pi}(\varphi(A) \circ \varphi(B))=\sigma_{\pi}(A B)$ for all $A, B \in \mathfrak{A}$, then it is linear, and either $\varphi$ or $-\varphi$ is multiplicative or anti-multiplicative. Therefore, either $\varphi$ or $-\varphi$ is an algebra isomorphism or anti-isomorphism.

Proof. According to Lemmas 2 and 4 the map $\varphi$ is a bijective linear operator between $\mathfrak{A}$ and $\mathfrak{B}$ and is of type either (a) or (b) (cf. Lemma 4).

If $\varphi$ is of type (a), then for any $x \in X$ and $f \in X^{*}, \varphi(x \otimes f)=C x \otimes D f$, where $C: X \rightarrow Y$ and $D: X^{*} \rightarrow Y^{*}$ are bijective linear operators. Now $\sigma_{\pi}((x \otimes f) \circ(x \otimes f))=\sigma_{\pi}\{(f(x) x) \otimes f\}=\left\{(f(x))^{2}\right\}$, but also

$$
\begin{aligned}
\sigma_{\pi}(\varphi(x \otimes f) \circ \varphi(x \otimes f)) & =\sigma_{\pi}((C x \otimes D f) \circ(C x \otimes D f)) \\
& =\sigma_{\pi}((((D f)(C x)) C x) \otimes D f)=\left\{((D f)(C x))^{2}\right\} .
\end{aligned}
$$

The peripheral multiplicativity of $\varphi$ implies that $(f(x))^{2}=((D f)(C x))^{2}$, and hence $(D f)(C x)= \pm f(x)$. For any $A \in \mathfrak{A}$ and $x \otimes f \in B_{1}(X)$ we have

$$
\begin{aligned}
\{f(A x)\} & =\sigma_{\pi}(A x \otimes f)=\sigma_{\pi}(A \circ(x \otimes f)) \\
& =\sigma_{\pi}(\varphi(A) \circ \varphi(x \otimes f))=\sigma_{\pi}(\varphi(A) \circ(C x \otimes D f)) \\
& \left.=\sigma_{\pi}((\varphi(A) C x) \otimes D f)\right)=\{(D f)(\varphi(A) C x)\} \\
& =\left\{(D f)\left(C C^{-1}(\varphi(A) C x)\right)\right\}=\left\{ \pm f\left(\left(C^{-1} \varphi(A)\right) C x\right)\right\} .
\end{aligned}
$$

Since this holds for every $f \in X^{*}$, it follows that $A x=\left( \pm C^{-1} \varphi(A) C\right) x$, i.e. $\pm \varphi(A) C x=C A x$. Hence, $\varphi(A) y= \pm\left(C A C^{-1}\right) y$ for any $y \in Y$, i.e. $\varphi(A) y=\tau_{A}(y)\left(C A C^{-1}\right) y=\tau_{A}(y) \psi(A) y$, where $\tau_{A}$ is a \pm 1 -valued function on $Y$ (possibly depending on $A$ ) and $\psi$ is the injective, linear, multiplicative operator from $\mathfrak{A}$ into $B(Y)$ defined by $\psi(A)=C A C^{-1}$. The linearity of $\varphi(A)$ and $\psi(A)$ implies that $\tau_{A}$ actually does not depend on $y$, thus $\varphi(A)=$ $\tau_{A} \psi(A)$, where $\tau_{A}$ takes values \pm 1 and possibly depends on $A$. The linearity of $\varphi$ and $\psi$ implies that $\tau_{A}$ does not depend on $A$ either. Hence $\varphi= \pm \psi$, i.e. either $\varphi$ or $-\varphi$ is multiplicative. 
If $\varphi$ is of type (b), then for any $x \in X$ and $f \in X^{*}, \varphi(x \otimes f)=E f \otimes F x$, where $E: X^{*} \rightarrow Y$ and $F: X \rightarrow Y^{*}$ are bijective linear operators. Now

$$
\begin{aligned}
\sigma_{\pi}(\varphi(x \otimes f) \circ \varphi(x \otimes f)) & =\sigma_{\pi}((E f \otimes F x) \circ(E f \otimes F x)) \\
& =\sigma_{\pi}(((F x)(E f) E f) \otimes F x)=\left\{((F x)(E f))^{2}\right\} .
\end{aligned}
$$

The peripheral multiplicativity yields $(f(x))^{2}=((F x)(E f))^{2}$, so $(F x)(E f)$ $= \pm f(x)$. For any $A \in \mathfrak{A}$ and $x \otimes f \in B_{1}(X)$ we have

$$
\begin{aligned}
\left\{\left(A^{*} f\right)(x)\right\} & =\{f(A x)\}=\sigma_{\pi}(A x \otimes f)=\sigma_{\pi}(A \circ(x \otimes f)) \\
& =\sigma_{\pi}(\varphi(A) \circ \varphi(x \otimes f))=\sigma_{\pi}(\varphi(A) \circ(E f \otimes F x)) \\
& =\sigma_{\pi}((\varphi(A) E f) \otimes F x)=\{(F x)(\varphi(A) E f)\} \\
& =\left\{(F x)\left(E E^{-1}(\varphi(A) E f)\right)\right\}=\left\{ \pm\left(E^{-1}(\varphi(A) E f)\right)(x)\right\},
\end{aligned}
$$

where $A^{*}: X^{*} \rightarrow X^{*}$ is the adjoint operator of $A$. Since this holds for every $x \in X$, it follows that $A^{*} f=\left( \pm E^{-1} \varphi(A) E\right) f$. Consequently, $A^{*}=$ $\pm E^{-1} \varphi(A) E$. Hence, $\pm \varphi(A) E=E A^{*}$, i.e. $\varphi(A)= \pm \eta(A)$, where $\eta$ is the injective, linear, anti-multiplicative operator from $\mathfrak{A}$ into $B(Y)$ defined by $\eta(A)=E A^{*} E^{-1}$, i.e. either $\varphi$ or $-\varphi$ is anti-multiplicative.

It is clear that the first case in Theorem 1 is possible only if $X$ and $Y$ are linearly isomorphic, while the second case is possible only if $X$ and $Y^{*}$ are linearly isomorphic. Note that the conclusion of Theorem 1 also holds if $\varphi$ satisfies equation (2) for each $A \in \mathfrak{A}$ and every operator $B \in B(X)$ of finite rank, or even of rank at most two. If $B \in B_{1}(X)$ then the equation $\sigma_{p}(\varphi(A) \circ \varphi(B))=\sigma_{p}(A B)$, similar to (2) and considered in [11], where $\sigma_{p}(A)$ is the point spectrum of $A$, implies (2), but not vice versa.

Since $X, Y$ are Banach spaces, under the hypotheses of Theorem 1 the bijections $C, E$ and their inverses are actually bounded linear operators by the Closed Graph Theorem. Consequently, $\varphi$ is also a bounded linear operator.

COROLlary 2. If a surjective map $\varphi$ between the algebras of compact operators (or of finite-rank operators) on $X$ and $Y$ is peripherally-multiplicative, then either $\varphi$ or $-\varphi$ is a continuous algebra isomorphism or antiisomorphism.

If, in addition to the hypotheses of Theorem 1 , there exists an $A_{0} \in \mathfrak{A}$ such that $\sigma_{\pi}\left(\varphi\left(A_{0}\right)\right) \neq-\sigma_{\pi}\left(A_{0}\right)$, then the case " $-\varphi$ " of Theorem 1 is ruled out. Indeed, if $\varphi=-\psi$, where $\psi(A)=C A C^{-1}$, then $\sigma_{\pi}\left(\varphi\left(A_{0}\right)\right)=$ $\sigma_{\pi}\left(-\psi\left(A_{0}\right)\right)=-\sigma_{\pi}\left(\psi\left(A_{0}\right)\right)=-\sigma_{\pi}\left(A_{0}\right)$, contradicting the assumption. Similar arguments apply in the case when $\varphi=-\eta$, where $\eta(A)=E A^{*} E^{-1}$. Hence $\varphi \neq-\psi,-\eta$, i.e. $\varphi=\psi$ or $\varphi=\eta$ and thus $\varphi$ is an algebra isomorphism or anti-isomorphism. In the first case there exists a linear isomorphism $C: X \rightarrow Y$ so that $\varphi(A)=C A C^{-1}$, while in the second case there exists a linear isomorphism $E: X^{*} \rightarrow Y$ so that $\varphi(A)=E A^{*} E^{-1}$. 
Consequently, we obtain the following theorem:

Theorem 2. Let $\varphi$ be as in Theorem 1. If, in addition, $\sigma_{\pi}\left(\varphi\left(A_{0}\right)\right) \neq$ $-\sigma_{\pi}\left(A_{0}\right)$ for some $A_{0} \in \mathfrak{A}$ then $\varphi$ is a bijective and bounded linear operator which is either multiplicative, in which case $X$ is isomorphic to $Y$, or antimultiplicative, in which case $X$ is isomorphic to $Y^{*}$.

Consequently, under the conditions of Theorem 2, if $X \not Y^{*}$ then $\varphi$ is multiplicative, hence an algebra isomorphism, while if $X \neq Y$, then $\varphi$ is anti-multiplicative, hence an algebra anti-isomorphism.

Clearly, Theorem 2 holds if there exists an $A_{0} \in \mathfrak{A}$ with $\sigma_{\pi}\left(A_{0}\right) \neq\{0\}$ such that $\sigma_{\pi}\left(\varphi\left(A_{0}\right)\right)=\sigma_{\pi}\left(A_{0}\right)$. In particular, we have:

Corollary 3. Let $\mathfrak{A} \subset B(X)$ and $\mathfrak{B} \subset B(Y)$ be unital standard operator algebras. If a peripherally-multiplicative surjective map $\varphi: \mathfrak{A} \rightarrow \mathfrak{B}$ is unital, then it is a continuous multiplicative or anti-multiplicative linear operator.

Note that if, under the hypotheses of Theorem $2, \mathfrak{A}$ is the algebra of finite rank operators on $X$ [resp. its closure], then $\mathfrak{B}$ is the algebra of finite rank operators on $Y$ [resp. its closure]. If $\mathfrak{A}$ is the algebra of compact operators on $X$, then the elements of $\mathfrak{B}$ are automatically compact operators on $Y$.

Acknowledgements. The authors would like to thank the referees for valuable suggestions to improve the manuscript and also Scott Lambert for helpful discussions and comments.

\section{References}

[1] Z. F. Bai and J. C. Hou, Characterizing isomorphisms between standard operator algebras by spectral functions, J. Operator Theory, 54 (2005), 291-303.

[2] Z. F. Bai, J. C. Hou and Z. B. Xu, Maps preserving numerical radius distance on $C^{*}$-algebras, Studia Math. 162 (2004), 97-104.

[3] S. A. Grigoryan and T. V. Tonev, Shift-Invariant Uniform Algebras on Groups, Monograf. Mat. 68, Birkhäuser, Basel, 2006.

[4] O. Hatori, T. Miura and H. Takagi, Characterizations of isometric isomorphisms between uniform algebras via non-linear range-preserving properties, Proc. Amer. Math. Soc. 134 (2006), 2923-2930.

[5] J. C. Hou and Q. H. Di, Maps preserving numerical ranges of operator products, ibid., 1435-1446.

[6] A. A. Jafarian and A. R. Sourour, Spectrum-preserving linear maps, J. Funct. Anal. 66 (1986), 255-261.

[7] K. Jarosz, When is a linear functional multiplicative?, in: Function Spaces, Contemp. Math. 232, Amer. Math. Soc., 1999, 201-210.

[8] S. Lambert, A. Luttman and T. Tonev, Weakly peripherally-multiplicative mappings between uniform algebras, in: Function Spaces, Contemp. Math. 435, Amer. Math. Soc., Providence, RI, 2007, 265-281. 
[9] C. K. Li and N. K. Tsing, Linear preserver problems: a brief introduction and some techniques, Linear Algebra Appl. 162-164 (1992), 217-235.

[10] A. Luttman and T. Tonev, Uniform algebra isomorphisms and peripheral multiplicativity, Proc. Amer. Math. Soc. 135 (2007), 3589-3598.

[11] L. Molnár, Some characterizations of the automorphisms of $B(H)$ and $C(X)$, ibid. 130 (2002), 111-120.

[12] -, Selected Preserver Problems on Algebraic Structures of Linear Operators and on Function Spaces, Lecture Notes in Math. 1895, Springer, Berlin, 2007.

[13] N. V. Rao and A. K. Roy, Multiplicatively spectrum-preserving maps of function algebras, Proc. Amer. Math. Soc. 133 (2005), 1135-1142.

[14] N. V. Rao, T. V. Tonev and E. T. Toneva, Uniform algebra isomorphisms and peripheral spectra, in: Topological Algebras and Applications, Contemp. Math. 427, Amer. Math. Soc., Providence, RI, 2007, 401-416.

[15] P. Šemrl, Two characterizations of automorphisms on $B(X)$, Studia Math. 105 (1993), 143-149.

Department of Mathematical Sciences

Division of Mathematics

The University of Montana

Missoula, MT 59812-1032, U.S.A.

E-mail: tonevtv@mso.umt.edu and Computer Science Box 5815, Clarkson University Potsdam, NY 13699, U.S.A. E-mail: aluttman@clarkson.edu

Received March 11, 2008

Revised version September 11, 2008 